\title{
A finite element and experimental analysis of durability tested springs
}

\author{
Ying Wang ${ }^{1}$, Constantinos Soutis ${ }^{1, *}$, and Lorenzo Gagliardi ${ }^{2}$ \\ ${ }^{1}$ Faculty of Science and Engineering, The University of Manchester, James Light Building, Manchester, M13NJ, UK \\ ${ }^{2}$ Jaguar Land Rover, Warwick, CV35 0RR, UK
}

\begin{abstract}
Ageing of vehicles has become a major concern as the vehicles reach the end of their original design life. Corrosion is part of long-term ageing and forms one of the critical degradation processes affecting the mechanical properties, such as stiffness, affecting the structural integrity and life of key components. In this study, a finite element and experimental analysis was carried out to investigate the response of corroded springs in relation to the morphology of a corroded surface. An FE model was created using ABAQUS that correlates the stiffness changes with ageing. The analysis of corrosion-induced stress is then performed to determine the stress fields within the corrosion region. The durability tested springs were analysed with a microscope to identify the morphology of corrosion pits; the measured pattern was adopted in the subsequent FE simulations. A spring test machine was employed to perform the mechanical tests. The load-deflection behaviour of durability tested springs was recorded and then was used to validate the FE simulations.
\end{abstract}

\section{Introduction}

\subsection{Background}

Ageing of vehicles has become a major concern as the vehicles reach the end of their original design life. Corrosion is part of long-term ageing and forms one of the critical degradation processes affecting the mechanical properties, such as stiffness, affecting the structural integrity and life of key components. When localised corrosion occurs, stiffness and strength reduction can be difficult to predict because of the complicated effects of uneven surfaces and uneven material properties on the stress fields. Significant work is being accomplished by the automotive industry in this area, with emphasis on widespread fatigue damage [1-6]. The present study, however, considers long-term ageing in the context of corrosion and its effect on the system behaviour. The purpose of this study is to develop a full predictive FE model for suspension springs that can simulate and predict the dynamic behaviour of corroded springs.

Fig. 1 shows an example of the mechanical response of a suspension spring under static loading. The forcedisplacement relationship shows that the spring stiffness (or spring rate) is a variable, bilinear rate that is approximated, as indicated by $k_{1}$ and $k_{2}$ (slope of the curve). The parameters $k_{1}$ and $k_{2}$ are referred to as static stiffness here.

The key dynamic characteristic of the suspension spring is the dynamic stiffness at prescribed preloads, amplitudes and frequencies. Basically, the stiffness is considered to be a design variable where an increased/decreased stiffness can affect the driveability and the driving experience in many different ways. Changing the stiffness and predicting the consequences of it is a complex and expensive process, and the result can in some cases be very hard to predict, especially in the presence of surface corrosion defects. It is important to understand the mechanical response of automotive helical springs containing surface corrosion.

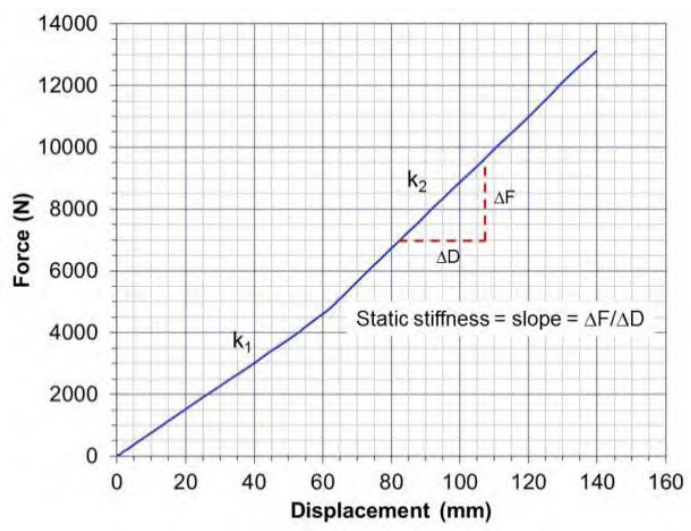

Fig. 1. Force-displacement curve of a suspension spring

Dynamic behaviour of isotropic and homogeneous helical springs has been widely studied in the past decades [1, 7-12], there are no in depth studies on the dynamic behaviour of a corroded suspension spring. For the corroded spring, the design formulas for helical compression springs are no longer suitable. In general, the geometry and loading encountered in three- 
dimensional corrosion problems is too complex for the stresses to be solved analytically. The stress calculation is further complicated because it is a function of the position along the wire, corrosion defect size and shape. Therefore, in this study we will use current available numerical analysis tools to perform the stress analysis.

In the present study, a nonlinear dynamic analysis is carried out to investigate the dynamic response of corroded springs in relation to corrosion depth, degree of surface corrosion and distributions. An FE model is created using ABAQUS that is used to correlate the stiffness changes of the corroded spring with corrosion depth and the degree of surface corrosion (DSC). The analysis of corrosion-induced stress is then performed.

\subsection{Dynamic stiffness}

The complex stiffness for a spring that is exposed to a sinusoidal displacement $x_{i}(t)$ with the corresponding output force $f_{\mathrm{o}}(t)$ is described as

$$
K^{*}=\frac{F_{O}^{*}}{X_{i}}=\frac{F_{O}}{X_{i}} \cos \delta+j \frac{F_{O}}{X_{i}} \sin \delta
$$

The displacement and the output force are given by $x_{i}(t)$ $=X_{i} e^{j \omega t}$ and $f_{\mathrm{o}}(t)=F_{o} e^{j(\omega t+\delta)}$, where $F_{\mathrm{o}}{ }^{*}=F_{\mathrm{o}} e^{j \omega t}$. $X_{i}$ is the displacement amplitude, $F_{o}$ is the force amplitude and $\delta$ is the phase angle between the input displacement and the output force, and $\omega$ is the input frequency.

A more general form of equation (1) can be expressed as:

$$
K^{*}=K^{\prime}+j K^{\prime \prime}
$$

where

$$
K^{\prime}=\frac{F_{O}}{X_{i}} \cos \delta \text { and } K^{\prime \prime}=\frac{F_{O}}{X_{i}} \sin \delta
$$

The dynamic stiffness $K_{d}$ is the magnitude of $K^{*}$

$$
K_{d}=\sqrt{\left(K^{\prime}\right)^{2}+\left(K^{\prime \prime}\right)^{2}}=\frac{F_{O}}{X_{i}}
$$

\section{Geometry of corrosion defects}

\subsection{Corrosion geometry and distribution}

Fig. 2(a) shows an example of the durability tested spring that contains several corrosion defects. It is difficult to generate an actual profile of corrosion that can be implemented in the FE simulation. For simplicity, the corroded surface is assumed to be smooth with a uniform corrosion depth as shown in Fig. 2(b). Therefore, the corrosion defect can be described in terms of the parameters ( $\alpha, \beta, D, r$ and $R$ ) shown in Fig. 2(c). The angle $\alpha$ is used to describe the region that is covered by the corrosion defect in the wire's cross-section $(0 \leq \alpha$ $\left.\leq 360^{\circ}\right), \beta$ is the angle covered by the corrosion defect in the coil's section $\left(0 \leq \beta \leq 180^{\circ}\right), D$ is the mean diameter of the corresponding coil, $r$ is the radius of corroded surfaces and $R$ is the radius of the spring wire. The depth of corrosion $(d)$ is given by:

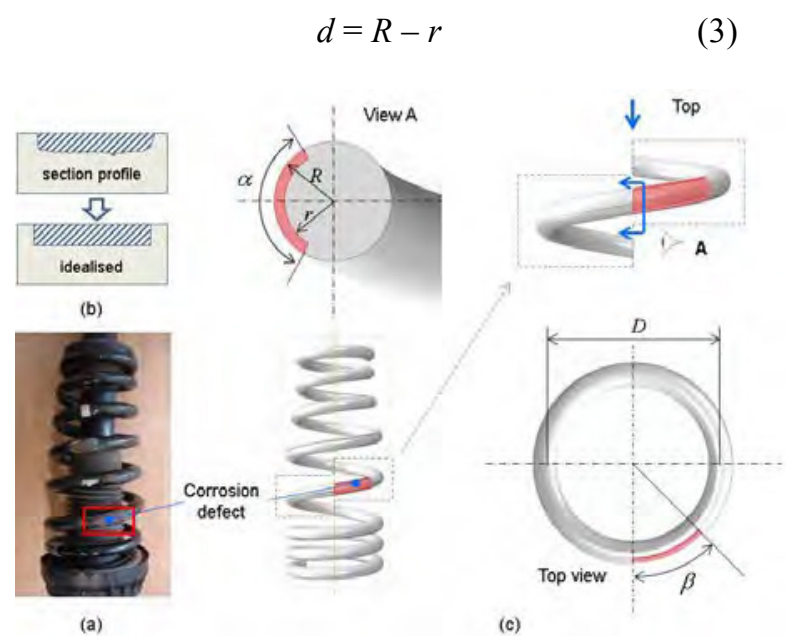

Fig. 2. Schematic showing: (a) the tested spring containing several corrosion defects; (b) the real and idealised surface profile of the section; (c) the geometry of the corrosion defect describing its associated geometrical parameters.

Fig. 3 demonstrates a serious of typical corrosion spatial distributions. For the single coil corrosion (e.g. coil 5), two patterns were selected for the modelling process: case A ( 1 in coil 5$)$ and case B ( 2 in coil 5$)$. The reason for choosing coil 5 is because the maximum stress is often seen in this coil. For the multi-coil corrosion case (coils 2 to 7), two further patterns were selected: case C ( 1 in coils $2-7)$ and case D (2 in coils $2-$ $7)$. The meaning of these patterns is that, the 1 in coil 5 represents that 1 corrosion defect exists in coil 5; the 2 in coil 5 represent that 2 corrosion defects exist in coil 5; the 1 in coils $2-7$ is that 1 corrosion defect exists in each coil for the coils 2 to 7 and the 2 in coils $2-7$ is that 2 corrosion defects exist in each coil for the coils 2 to 7 .

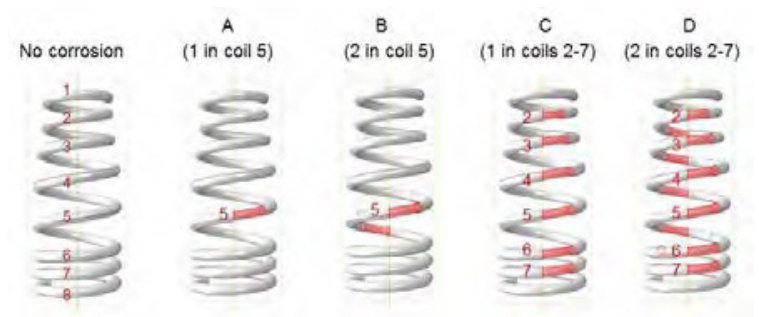

Fig. 3. Corrosion spatial distributions: A (1 in coil 5); B (2 in coil 5); C (1 in coils 2-7) and D (2 in coils 2-7).

\subsection{Degree of surface corrosion}

The degree of surface corrosion (DSC) was used to correlate the dynamic stiffness change of a corroded spring with corrosion surface area. The DSC is defined as the ratio (as a percentage) of the area of the corroded surfaces to the original surface area of the spring and is given by the following expression:

$$
D S C=\frac{1}{A_{0}} \sum_{i=1}^{n} A_{i} \times 100 \%
$$

where $n$ is the number of corrosion defects, $A_{\mathrm{i}}$ is the surface area of the $i^{\text {th }}$ corrosion defect, $A_{0}$ is the original 
surface area. $A_{0}=2 \pi R l_{t}, R$ and $l_{t}$ are the radius and length of spring wire. In the FE model, the size of each corrosion defect can be obtained by changing angles ( $\alpha$ or/and $\beta$ ) to get different combinations.

\section{FE modelling}

\subsection{Description of FE model}

The FE model was created using ABAQUS to simulate the dynamic behaviour of a helical compression spring. The spring CAD drawing (stp format) provided by JLR was imported into ABAQUS and then this imported file was edited using ABAQUS CAE to create the FE model of a corroded spring. The geometric parameters are determined in terms of the corrosion geometry model described above. Different corrosion geometries are selected to study its effect on the dynamic behaviour.

An 8-node brick element (C3D8) was used in the model. Approximately 65000-80000 elements were used in total, depending on the depth of corrosion and its distribution on the surface. Fig. 4 demonstrates the overview of the mesh and detailed view of a mesh at corrosion defect regions, respectively.
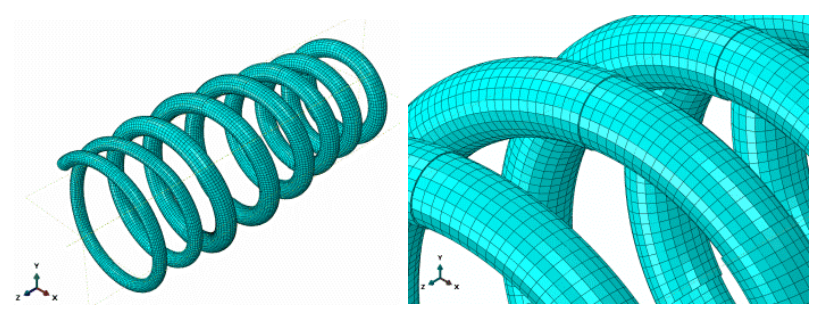

Fig. 4. Diagrams showing overview of mesh and detailed view of a mesh at the corrosion regions.

The spring material is a high-strength martensitic steel (54SiCr6). The isotropic material model was adopted; the elastic modulus and Poisson's ratios are assumed to be equal to $200 \mathrm{GPa}$ and 0.28 , respectively. The yield stress is $1680 \mathrm{MPa}$ and is used in the FE model. For the elastic-plastic deformation, the true stress $(\sigma)$ - true strain $(\varepsilon)$ relationship of the spring material, provided by supplier, can be expressed as follow.

$$
\sigma=2430 \varepsilon^{0.08}(0.0085 \leq \varepsilon \leq 0.6)
$$

\subsection{Boundary conditions}

The boundary condition for the FE model is shown in Fig. 5, in which one end of the spring is fixed and the load is applied from the other end. The load was applied using a "dummy node" which was specified at the axis of the coils. Every node in the contact region at the top of the coil is constrained to have the same displacement as the dummy node, via a linear multi-point constraint EQUATION available in ABAQUS [13].

The prescribed displacement describes the movement of the spring which occurs during driving. A static preload (e.g. $77 \mathrm{~mm}$ displacement) was applied first and then a dynamic load was superimposed from the other end to record the dynamic response for each frequency applied (see Fig. 5).

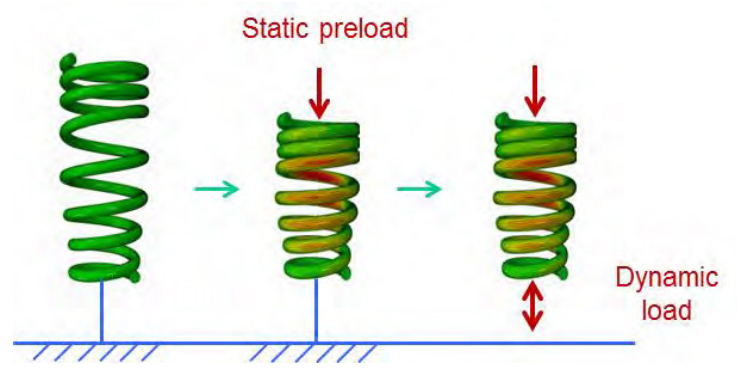

Fig. 5. Boundary conditions applied in the FE model.

The dynamical simulations were carried out in several steps, assigned with an individual displacement amplitude curve with a corresponding frequency, see Fig. 6. A sinusoidal displacement was assumed with the displacement amplitude of $5 \mathrm{~mm}$. The increment size was adjusted for each step, since a higher frequency requires a smaller time step size in order to achieve high accuracy and for being able to capture the peaks in the time response. Fig. 7 shows an FE simulation example of the reaction force at the corresponding frequency, which indicates that the force increases with increased frequency.

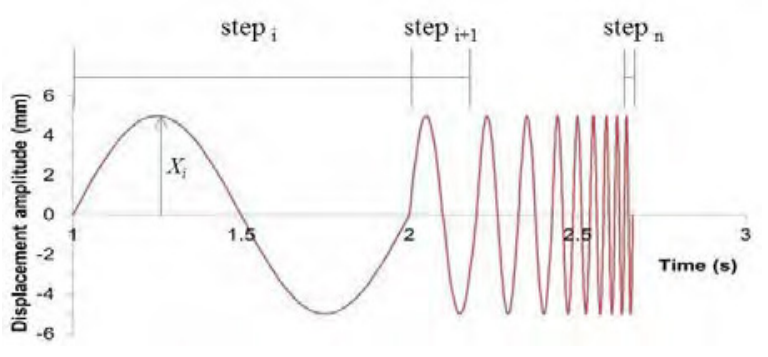

Fig. 6. Frequency step

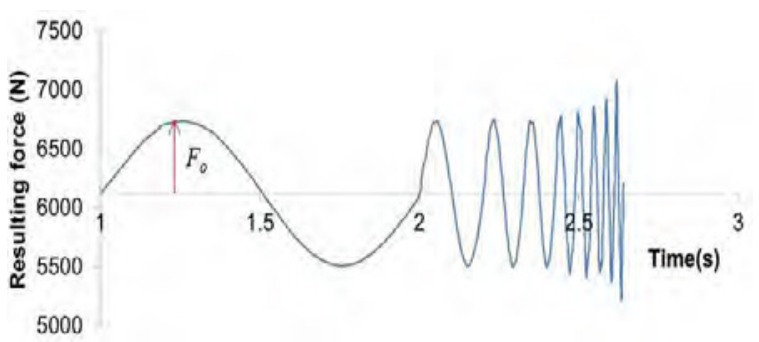

Fig. 7. Reaction force at corresponding frequency

Surface to surface contact is defined when the surfaces of the wire come into contact with each other. At this point simple Coulomb friction is introduced between coils. The coil contact is modelled using a hard contact-penalty algorithm provided by the ABAQUS/Standard finite element program. The frictional coefficient for surfaces in contact is assumed to be 0.5 . 


\section{Dynamic stiffness changes due to corrosion}

\subsection{Influence of corrosion depth}

Consider single coil corrosion (Case A) and let $\alpha=360^{\circ}$ and $\beta=45^{\circ}$. Dynamic stiffness is plotted against frequency for different corrosion depth shown in Fig. 8. As can be seen from the figure, the dynamic stiffness is moderately increased with an increase in frequency, if the frequency is below approximately $8 \mathrm{~Hz}$. The increase in dynamic stiffness becomes larger when the frequency is more than $10 \mathrm{~Hz}$. For other cases (Case B, C and D), the dynamic stiffness changes show a similar pattern but the magnitude of the stiffness is slightly different.

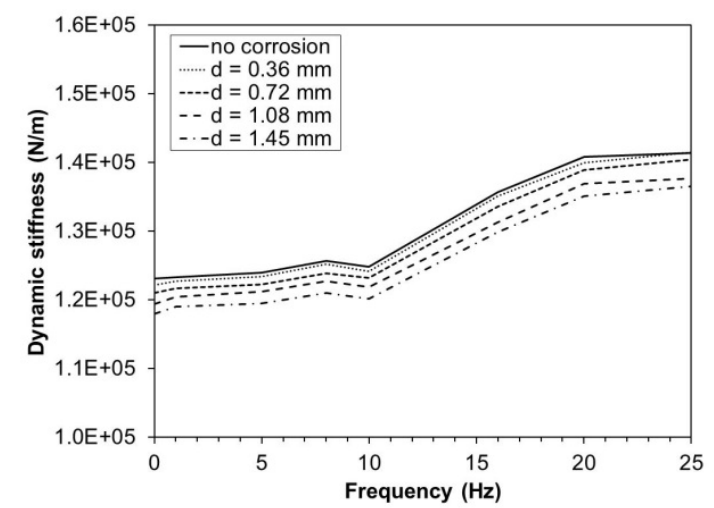

Fig. 8. Stiffness against frequency at different corrosion depth.

The dynamic stiffness at different frequencies is replotted against corrosion depth, along with a regression fit, shown in Fig. 9. The $R^{2}$ value for the regression lines is 0.996 for all cases. It can be seen that the dynamic stiffness is decreased as the corrosion depth increases for all the range of frequencies. The static stiffness (the case of frequency $=0$ ) is also plotted, indicated by blue line.

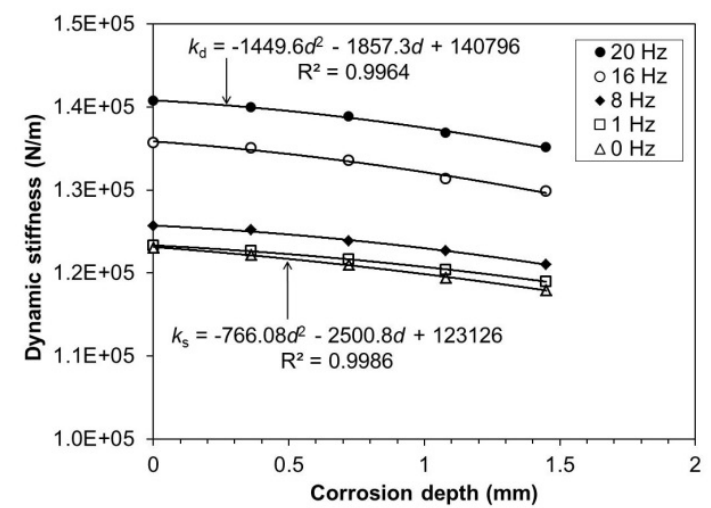

Fig. 9. Stiffness against corrosion depth at different frequencies

\subsection{Influence of DSC}

The dynamic stiffness is plotted against the degree of surface corrosion (DSC), along with a regression fit for each, shown in Fig. 10. The $R^{2}$ value for the regression lines is 0.999 for all. Two regression equations, for frequency $=0 \mathrm{~Hz}$ (static) and frequency $=25 \mathrm{~Hz}$, are shown in the figure, which can be used to predict the dynamic stiffness of the spring with different DSC. The trend for all the cases is very similar compared to the static. The only difference is the magnitude of the dynamic stiffness, depending on the frequency

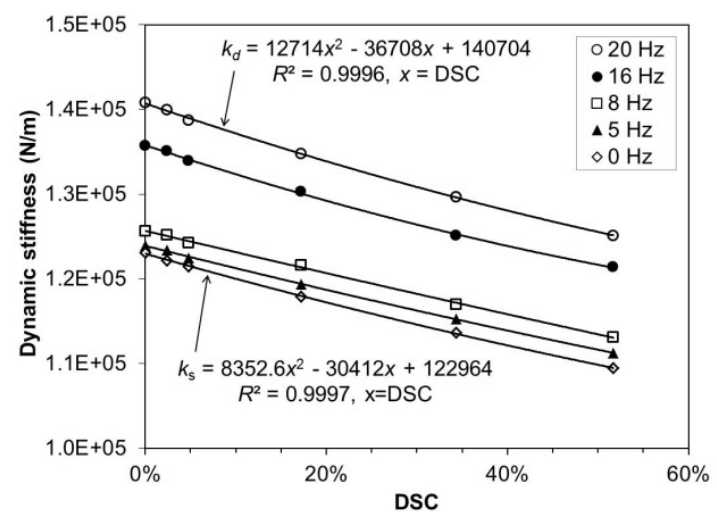

Fig. 10. Dynamic stiffness against DSC at different frequency.

\section{Analysis of corrosion-induced stress}

\subsection{Uniform corrosion}

The uniform corrosion observed on the tested spring was idealised as a smooth surface with a uniform corrosion depth in order to perform the FE analysis, as described in Section 2 (see Fig. 2). The stress distribution on the surface is significantly changed due to the corrosion defects. Fig. 11 shows an example of stress distributions in the presence of corrosion defects.

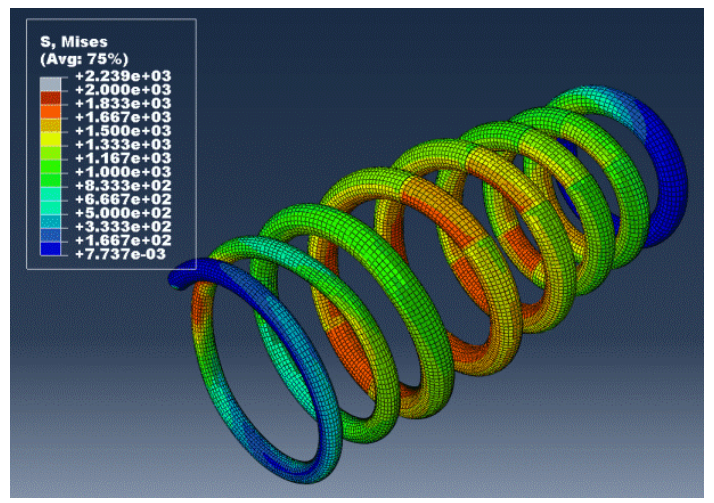

Fig. 11. Stress contours of the spring with surface corrosion $\left(d=0.36 \mathrm{~mm}, \alpha=360^{\circ}\right.$ and $\left.\beta=45^{\circ}\right)$.

Fig. 12 shows the history of von Mises stress at different frequency steps. The stress was measured at the location indicated by a symbol ' + '. As can be seen from the figure, the stress is moderately increased with an increase in frequency while the corrosion depth shows a significant effect on the stress.

The maximum von Mises stresses at each frequency are plotted against corrosion depth in Fig. 13. This indicates that the von Mises stress is increased with the increase of corrosion depth. 


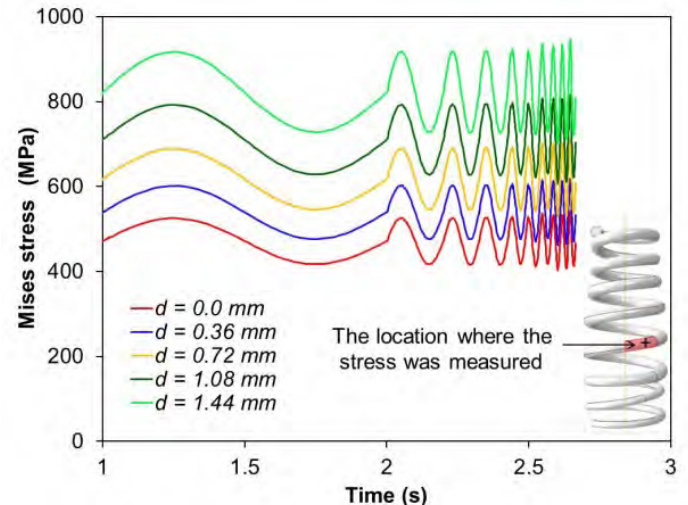

Fig. 12. Von Mises stress history plot at different corrosion depth (Case A, $\alpha=360^{\circ}, \beta=45^{\circ}$ )

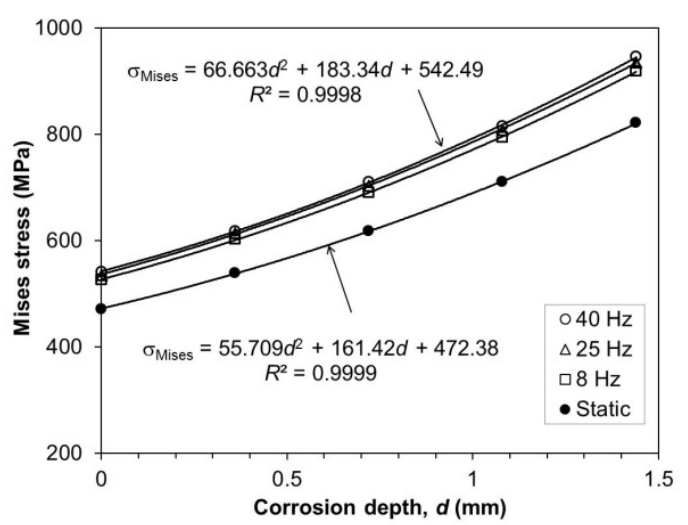

Fig. 13. Plot of maximum stresses at each frequency against corrosion depth.

Fig. 14 shows the effect of the degree of surface corrosion (DSC) on the magnitude and distribution of stresses. The von Mises stress was measured at the same position for each case, as indicated in the previous diagram (Figure 12). It is assumed that $\alpha$ is $360^{\circ}$ and corrosion depth is $0.36 \mathrm{~mm}$. The angle $\beta$ is variable so that different DSC values can be obtained. As can be seen from the figure, the von Mises stress is gradually decreased with an increase in DSC.

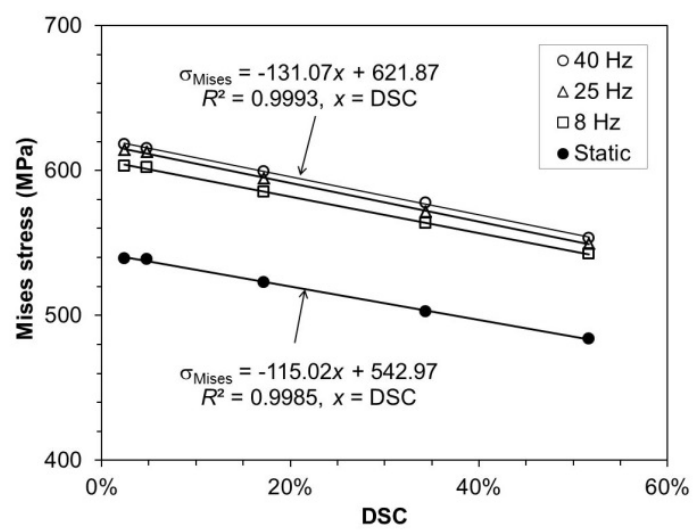

Fig. 14. Von Mises stress against DSC at different frequency.

\subsection{Pitting corrosion}

Fig. 15 shows the morphology of a corroded surface of the durability tested spring. The corrosion pits are randomly distributed within the corrosion region and the radius is between 0.1 and $0.3 \mathrm{~mm}$, approximately. The distributions of these pits are idealised into two patterns and then pattern 1 was adopted in the subsequent $\mathrm{FE}$ simulations.
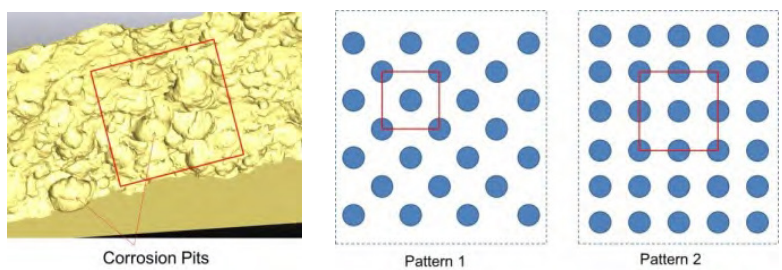

Fig. 15. The morphology of corroded surface observed on tested springs and two idealised distribution patterns.

A multiscale FE model was developed to determine the stress on these corrosion pits. Fig. 16 demonstrates how the FE model is built up. A full model is set up first; the sub-model is extracted from the full model using ABAQUS sub-model function $(1 / 4$ of coil, $8 \times 8 \times 6 \mathrm{~mm}$ and $2 \times 2 \times 1.5 \mathrm{~mm}$ sub-models are selected) and then the hemispherical pits are added to the smallest one. An 8node linear brick element (C3D8) is adopted in the full spring model and all sub-models. A 10-node quadratic tetrahedron element (C3D10) was used in the model with the pits (e.g. 2 pits, 8 pits and 32 pits model).

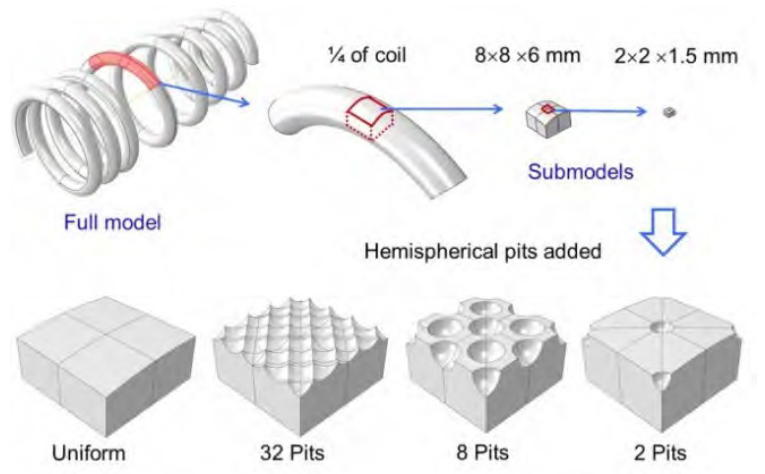

Fig. 16. Schematic showing the development of multiscale FE model for a spring with corrosion pits.

The FE simulation result is shown in Fig. 17, where the von Mises stress is measured at the bottom of the central pit, frequency is at $25 \mathrm{~Hz}$, the static preload is $6000 \mathrm{~N}$ and displacement amplitude is $5 \mathrm{~mm}$. The static (frequency $=0$ ) is also plotted to compare. As can be seen from the diagram, the worst case is 2 pits with the highest von Mises stress of $1368 \mathrm{MPa}$. The stress is decreased as the number of pits increases. The stress is $1087 \mathrm{MPa}$ for 8 pits, $942 \mathrm{MPa}$ for 32 pits and $748 \mathrm{MPa}$ for uniform, respectively. The stress contours for each case are shown in Fig. 18. 


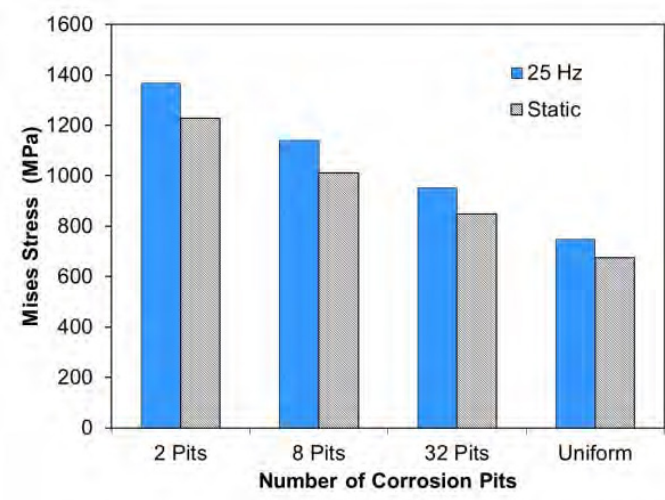

Fig. 17. Von Mises stress at the bottom of corrosion pits for the frequency of $25 \mathrm{~Hz}$.

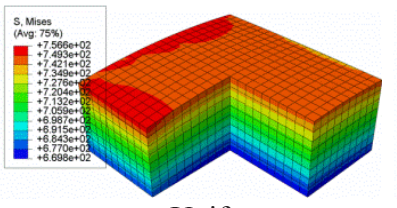

Uniform

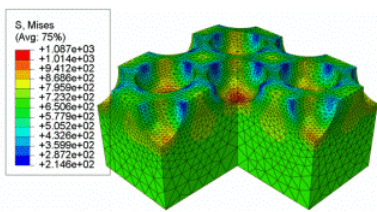

8 Pits

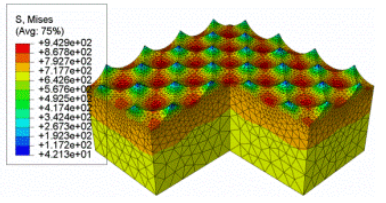

32 Pits

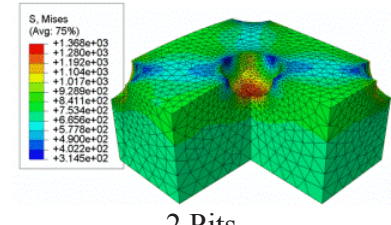

2 Pits
Fig. 18. Von Mises stress contours for $25 \mathrm{~Hz}$.

\subsection{Stress distribution with the coils}

The stresses were measured at the points 2, 3, 4 and 5 on the surface (indicated by a symbol ' + '), as shown in Fig. 19. The measured maximum von Mises stress at each corresponding frequency is plotted against frequency to determine the effect of frequency. As can be seen from the figure, the stress almost stays in the same level until the frequency reaches $20 \mathrm{~Hz}$, but afterwards the stress at the point 5 (coil 5) is slightly increased with an increased frequency while the stress at other points (coils 2-4) is decreased as the frequency increases.

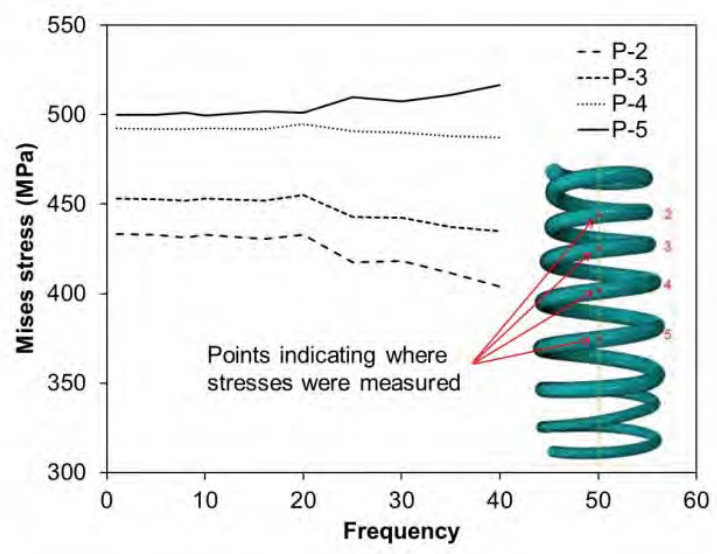

Fig. 19 Mises against frequency for each measured point.

\section{Experimental measurements}

Tests were conducted using a spring testing machine to validate the FE simulation results. The tests were defined by a series of steps, each step containing load, loading rate and data recording. The computer is connected via the serial port to the control interface. Test data is stored on the hard disc. The tests were performed at a room temperature.

The durability tested springs were analysed with a microscope to identify the surface condition. Small and shallow pits were found in few locations and the majority of the surface was in a very good condition. Therefore, the overall condition of the durability tested springs can be regarded as a nearly new spring.

Fig. 20 shows a comparison of FE simulations and experimental tests. It can be seen from the diagram, the mechanical responses from the FE simulation is very close to that from the experimental measurement. These observations confirmed the validity of the FE model.

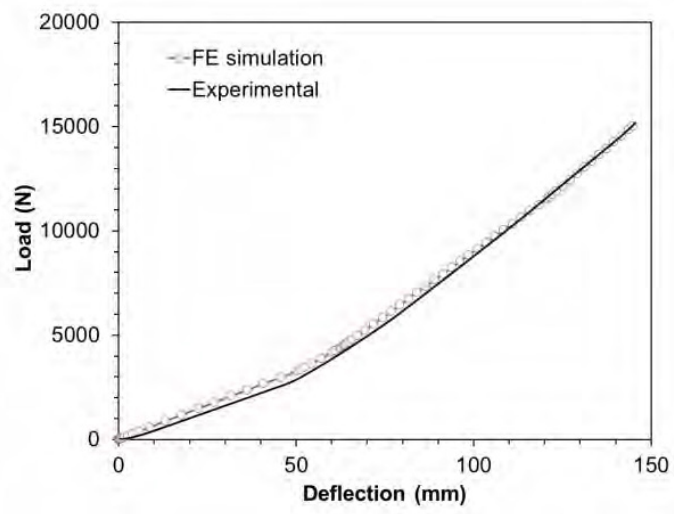

Fig. 20. Comparison of FE simulation and compression test.

\section{Concluding remarks}

A predictive FE model was developed which can simulate the dynamic response of springs with various corrosion defects. The model can be used to determine the stress/strain distribution in the presence of corrosion defects.

The dynamic characteristic for an uncorroded spring is similar to that of the corroded spring for the frequencies studied here. For example, the trend of dynamic stiffness changes for all selected cases is very similar; the only difference is the magnitude of the dynamic stiffness, depending on the frequency.

Dynamic stiffness $\left(K_{d}\right)$ is increased with an increased frequency for all cases while the $K_{d}$ is decreased with the increase of either corrosion depth or DSC.

The surface stress is significantly influenced by the morphology of corroded surfaces, corrosion depth and frequency. The stress on the corroded surface increases with the increase of corrosion depth.

\section{Acknowledgements}


This work was supported by Jaguar Land Rover and the UKEPSRC grant EP/L025752/1 as part of the jointly funded Programme for Simulation Innovation.

\section{References}

1. A.M. Wahl, Mechanical Springs. (1963)

2. Y. Prawoto, M. Ikeda, S.K. Manville, and A. Nishikawa, Eng Fail Anal. 15(8):1155-1174 (2008)

3. B. Pyttel, I. Brunner, B. Kaiser, C. Berger, and M. Mahendran, Int J Fatigue. 60:101-109 (2014)

4. M.A. Zaccone, Practical Failure Analysis. 1(3):51$62(2001)$

5. Y. Yamada, Materials for Springs. (2007)

6. M.T. Todinov, Int J Mech Sci. 41:357-370 (1999)

7. L.E. Beckera, G.G. Chassieb, and W.L. Cleghornb, Int J Mech Sci. 44:825-841 (2002)

8. C.L. Dym, J Mech Design. 131(7):071004:1-5 (2009)

9. J.E. Mottershead, International Journal of Mechanical Sciences. 22(5):267-283 (1980)

10. D. Pearson, J Mech Eng Sci. 24(4):163-171 (1982)

11. M. Takata, F. Dammak, S. Abid, and M. Haddar, J Mech Mater Struct. 3(4):641-658 (2008)

12. V. Ylldırım, Int J Mech Sci. 115-116:280-298 (2016)

13. ABAQUS Aanalysis User's Mannul (2016) 